\title{
On a Negative Flow of the AKNS Hierarchy and Its Relation to a Two-Component Camassa-Holm Equation ${ }^{\star}$
}

\author{
Henrik ARATYN ${ }^{\dagger}$ and Jose Francisco GOMES $\ddagger$ and Abraham H. ZIMERMAN $\ddagger$ \\ $\dagger$ Department of Physics, University of Illinois at Chicago, \\ 845 W. Taylor St., Chicago, Illinois 60607-7059 \\ E-mail: aratyn@uic.edu \\ ¥ Instituto de Física Teórica-UNESP, Rua Pamplona 145, 01405-900 São Paulo, Brazil \\ E-mail: jfg@ift.unesp.br, zimerman@ift.unesp.br
}

Received September 13, 2006, in final form October 05, 2006; Published online October 17, 2006

Original article is available at http://www.emis.de/journals/SIGMA/2006/Paper070/

\begin{abstract}
Different gauge copies of the Ablowitz-Kaup-Newell-Segur (AKNS) model labeled by an angle $\theta$ are constructed and then reduced to the two-component Camassa-Holm model. Only three different independent classes of reductions are encountered corresponding to the angle $\theta$ being $0, \pi / 2$ or taking any value in the interval $0<\theta<\pi / 2$. This construction induces Bäcklund transformations between solutions of the two-component Camassa-Holm model associated with different classes of reduction.
\end{abstract}

Key words: integrable hierarchies; Camassa-Holm equation; Bäcklund transformation

2000 Mathematics Subject Classification: 37K10; 35Q53; 53A07; 53B50

\section{Introduction}

It is widely known that the standard integrable hierarchies can be supplemented by a set of commuting flows of a negative order in a spectral parameter [1]. A standard example is provided by the modified KdV-hierarchy, which can be embedded in a new extended hierarchy. This extended hierarchy contains in addition to the original modified KdV equation also the differential equation of the sine-Gordon model realized as the first negative flow $[2,3,4,5,6,7]$.

Quite often the negative flows can only be realized in a form of non-local integral differential equations. The cases where the negative flow can be cast in form of local differential equation which has physical application are therefore of special interest. Recently in [11], a negative flow of the extended AKNS hierarchy [8] was identified with a two-component generalization of the Camassa-Holm equation. The standard Camassa-Holm equation [9, 10]

$$
u_{t}-u_{t x x}=-3 u u_{x}+2 u_{x} u_{x x}+u u_{x x x}-\kappa u_{x}, \quad \kappa=\text { const }
$$

enjoys a long history of serving as a model of long waves in shallow water. The two-component extension $[11,13]$ differs from equation (1.1) by presence on the right hand side of a new term $\rho \rho_{x}$, with the new variable $\rho$ obeying the continuity equation $\rho_{t}+(u \rho)_{x}=0$. Such generalization was first encountered in a study of deformations of the bihamiltonian structure of hydrodynamic type [12]. Various multi-component generalizations of the Camassa-Holm model have been subject of intense investigations in recent literature $[14,15,16,17,18]$.

\footnotetext{
${ }^{\star}$ This paper is a contribution to the Proceedings of the O'Raifeartaigh Symposium on Non-Perturbative and Symmetry Methods in Field Theory (June 22-24, 2006, Budapest, Hungary). The full collection is available at http://www.emis.de/journals/SIGMA/LOR2006.html
} 
A particular connection between extended AKNS model and a two-component generalization of the Camassa-Holm equation was found in [11] and in [13]. It was pointed out in [19] that the second order spectral equation for a two-component Camassa-Holm model can be cast in form of the first order spectral equation which after appropriate gauge transformations fits into an $s l(2)$ setup of linear spectral problem and associated zero-curvature equations.

The goal of this article is to formulate a general scheme for connecting an extended AKNS model to a two-component Camassa-Holm model which would encompass all known ways of connecting the solution $f$ of the latter model to variables $r$ and $q$ of the former model. Our approach is built on making gauge copies of an extended AKNS model labeled by angle $\theta$ belonging to an interval $0 \leq \theta \leq \pi / 2$ and then by elimination of one of two components of the $\operatorname{sl}(2)$ wave function reach a second order non-linear partial differential equation which governs the two-component Camassa-Holm model. We found that the construction naturally decomposes into three different classes depending on whether angle $\theta$ belongs to an interior of interval $0 \leq \theta \leq \pi / 2$ or is equal to one of two boundary values unifying therefore the results of [11] and [20]. The map between these three cases induces a Bäcklund like transformations between different solutions $f$ of the two-component Camassa-Holm equation.

\section{A simple derivation of a relation between AKNS and two-component Camassa-Holm models}

Our starting point is a standard first-order linear spectral problem of the AKNS model:

$$
\Psi_{y}=\left(\lambda \sigma_{3}+\mathcal{A}_{0}\right) \Psi=\lambda\left[\begin{array}{cc}
1 & 0 \\
0 & -1
\end{array}\right] \Psi+\left[\begin{array}{ll}
0 & q \\
r & 0
\end{array}\right] \Psi,
$$

where $\lambda$ is a spectral parameter, $y$ a space variable and $\Psi$ a two-component object:

$$
\Psi=\left[\begin{array}{l}
\psi_{1} \\
\psi_{2}
\end{array}\right]
$$

In addition, the system is augmented by a negative flow defined in terms of a matrix, which is inverse proportional to $\lambda$ :

$$
\Psi_{s}=D^{(-1)} \Psi=\frac{1}{\lambda}\left[\begin{array}{cc}
A & B \\
C & -A
\end{array}\right] \Psi .
$$

The compatibility condition arising from equations (2.1) and (2.3):

$$
\left(\mathcal{A}_{0}\right)_{s}-D_{y}^{(-1)}+\left[\lambda \sigma_{3}+\mathcal{A}_{0}, D^{(-1)}\right]=0
$$

has a general solution:

$$
D^{(-1)}=\frac{1}{4 \beta \lambda} M_{0} \sigma_{3} M_{0}^{-1}, \quad \mathcal{A}_{0}=M_{0 y} M_{0}^{-1}
$$

in terms of the zero-grade group element, $M_{0}$, of $\mathrm{SL}(2)$. Note that the solution, $D^{(-1)}$, of the compatibility condition is connected to $(1 / \lambda) \sigma_{3}$-matrix by a similarity transformation.

The factor $1 / 4 \beta$ in $(2.5)$ is a general proportionality factor which implies a determinant formula:

$$
A^{2}+B C=\frac{1}{16 \beta^{2}}
$$

for the matrix elements of $D^{(-1)}$. 
From (2.4) we find that

$$
\begin{aligned}
\left(\operatorname{Tr}\left(\mathcal{A}_{0}^{2}\right)\right)_{s} & =2 \operatorname{Tr}\left(\mathcal{A}_{0} \mathcal{A}_{0 s}\right)=-2 \operatorname{Tr}\left(\mathcal{A}_{0}\left[\lambda \sigma_{3}, D^{(-1)}\right]\right)=2 \operatorname{Tr}\left(\lambda \sigma_{3}\left[\mathcal{A}_{0}, D^{(-1)}\right]\right) \\
& =2 \operatorname{Tr}\left(\lambda \sigma_{3} D_{y}^{(-1)}\right)=4 A_{y}
\end{aligned}
$$

or

$$
A_{y}=\frac{1}{2}(r q)_{s} .
$$

When projected on the zero and the first powers of $\lambda$ the compatibility condition (2.4) yields

$$
q_{s}=-2 B, \quad r_{s}=2 C,
$$

and

$$
A_{y}=q C-r B, \quad B_{y}=-2 A q, \quad C_{y}=2 A r,
$$

respectively. Note that the first of equations (2.9) together with equations (2.8) reproduces formula (2.7).

Combining the above equations we find that

$$
A=-\frac{B_{y}}{2 q}=\frac{q_{s y}}{4 q}=\frac{C_{y}}{2 r}=\frac{r_{s y}}{4 r} .
$$

The spectral equation (2.1) reads in components:

$$
\psi_{1 y}=\lambda \psi_{1}+q \psi_{2}, \quad \psi_{2 y}=-\lambda \psi_{2}+r \psi_{1} .
$$

Now we eliminate the wave-function component $\psi_{2}$ by substituting

$$
\psi_{2}=\frac{1}{q}\left(\psi_{1 y}-\lambda \psi_{1}\right)
$$

into the remaining second equation of (2.11). In this way we obtain for $\psi_{1}$

$$
\psi_{1 y y}-\frac{q_{y}}{q} \psi_{1 y}+\frac{\lambda q_{y}}{q} \psi_{1}-\lambda^{2} \psi_{1}-r q \psi_{1}=0 .
$$

Introducing

$$
\psi=e^{-\int p \mathrm{~d} y} \psi_{1}
$$

with the integrating factor

$$
p(y)=\frac{1}{2}(\ln q)_{y}
$$

allows to eliminate the term with $\psi_{1 y}$ and obtain

$$
\psi_{y y}=\left(\lambda^{2}-\lambda(\ln q)_{y}-Q\right) \psi
$$

with

$$
Q=\frac{1}{2}(\ln q)_{y y}-\frac{1}{4}(\ln q)_{y}^{2}-r q=\frac{q_{y y}}{2 q}-\frac{3}{4}\left(\frac{q_{y}}{q}\right)^{2}-r q
$$

as in [20]. 
Eliminating $\psi_{2}$ from equation (2.3) yields for $\psi$ the following equation:

$$
\psi_{s}=\frac{1}{4 \lambda}\left(\frac{q_{s}}{q}\right)_{y} \psi-\frac{1}{2 \lambda} \frac{q_{s}}{q} \psi_{y}
$$

Compatibility equation $\psi_{\text {yys }}-\psi_{\text {syy }}=0$ yields

$$
\left(\frac{q_{s y}}{4 q}\right)_{y}=\frac{1}{2}(r q)_{s}
$$

in total agreement with (2.7). To eliminate $r$ from (2.16) we use that

$$
r=\frac{-A_{y}+q C}{B}
$$

as follows from the first equation from (2.9). Replacing $C$ by $1 /\left(B 16 \beta^{2}\right)-A^{2} / B$ as follows from the determinant relation (2.6) and recalling that $B=-q_{s} / 2$ according to equation (2.8) we obtain after substituting $r$ from (2.17) into (2.16):

$$
\left(\frac{q_{s y}}{q}\right)_{y}=\left(\frac{q_{s y y}}{q_{s}}-\frac{q_{s y} q_{y}}{q q_{s}}+\frac{1}{2 \beta^{2}} \frac{q^{2}}{q_{s}^{2}}-\frac{q_{s y}^{2}}{2 q_{s}^{2}}\right)_{s} .
$$

Note that alternatively we could have eliminated $q$ from equation

$$
\left(\frac{r_{s y}}{4 r}\right)_{y}=\frac{1}{2}(r q)_{s}
$$

and obtained an equation for $r$ only. It turns out that the equation for $r$ follows from equation (2.18) by simply substituting $r$ for $q$.

For brevity we introduce, as in [20], $f=\ln q$. Then expression (2.18) becomes:

$$
\left(f_{s} f_{y}\right)_{y}=-\left(\frac{f_{y}^{2}}{2}+\frac{f_{s y}^{2}}{2 f_{s}^{2}}-\frac{1}{2 \beta^{2} f_{s}^{2}}-\frac{f_{s y y}}{f_{s}}\right)_{s} .
$$

The above relation can be cast in an equivalent form:

$$
\frac{f_{s s}}{2 \beta^{2} f_{s}^{3}}+f_{s y} f_{y}+\frac{1}{2} f_{s} f_{y y}-\frac{f_{s s y y}}{2 f_{s}}+\frac{f_{s s y} f_{s y}}{2 f_{s}^{2}}+\frac{f_{s s} f_{s y y}}{2 f_{s}^{2}}-\frac{f_{s s} f_{s y}^{2}}{2 f_{s}^{3}}=0,
$$

which first appeared in [11]. The relation (2.20) is also equivalent to the following condition

$$
\left(\frac{1}{f_{s}}\right)_{s}=\beta^{2}\left(f_{s}^{2} f_{y}-f_{s s y}+\frac{f_{s s} f_{s y}}{f_{s}}\right)_{y}
$$

For a quantity $u$ defined as:

$$
u=\beta^{2}\left(f_{s}^{2} f_{y}-f_{s s y}+\frac{f_{s s} f_{s y}}{f_{s}}\right)-\frac{1}{2} \kappa
$$

with $\kappa$ being an integration constant, it holds from relation (2.21) that

$$
u_{y}=\left(\frac{1}{f_{s}}\right)_{s} .
$$


Next, as in [21], we define a quantity $m$ as $\beta^{2} f_{s}^{2} f_{y}$ and derive from relations (2.22) and (2.23) that

$$
\begin{aligned}
m & =\beta^{2} f_{s}^{2} f_{y}=u+\beta^{2}\left(f_{s s y}-\frac{f_{s s} f_{s y}}{f_{s}}\right)+\frac{1}{2} \kappa=u-\beta^{2} f_{s}\left(f_{s}\left(\frac{1}{f_{s}}\right)_{s}\right)_{y}+\frac{1}{2} \kappa \\
& =u-\beta^{2} f_{s}\left(f_{s} u_{y}\right)_{y}+\frac{1}{2} \kappa .
\end{aligned}
$$

Taking a derivative of $m$ with respect to $s$ yields

$$
\begin{aligned}
m_{s} & =\beta^{2}\left(2 f_{y} f_{s} f_{s s}+f_{s}^{2} f_{s y}\right)=2 m \frac{f_{s s}}{f_{s}}+\beta^{2} f_{s}^{2} f_{s y}=-2 m f_{s}\left(\frac{1}{f_{s}}\right)_{s}+\beta^{2} f_{s}^{2} f_{s y} \\
& =-2 m f_{s} u_{y}+\beta^{2} f_{s}^{2} f_{s y} .
\end{aligned}
$$

In terms of quantities $u$ and $\rho=f_{s}$ equations (2.23) and (2.25) take the following form

$$
\begin{aligned}
& \rho_{s}=-\rho^{2} u_{y}, \\
& m_{s}=-2 m \rho u_{y}+\beta^{2} \rho^{2} \rho_{y},
\end{aligned}
$$

for $m$ given by

$$
m=u-\beta^{2} \rho\left(\rho u_{y}\right)_{y}+\frac{1}{2} \kappa .
$$

An inverse reciprocal transformation $(y, s) \mapsto(x, t)$ is defined by relations:

$$
F_{x}=\rho F_{y}, \quad F_{t}=F_{s}-\rho u F_{y}
$$

for an arbitrary function $F$. Equations (2.26), (2.27) and (2.28) take a form

$$
\begin{aligned}
& \rho_{t}=-(u \rho)_{x}, \\
& m_{t}=-2 m u_{x}-m_{x} u+\beta^{2} \rho \rho_{x}, \\
& m=u-\beta^{2} u_{x x}+\frac{1}{2} \kappa
\end{aligned}
$$

in terms of the $(x, t)$ variables. Equation $(2.30)$ is called the compatibility condition, while equation (2.31) is the two-component Camassa-Holm equation [11], which agrees with standard Camassa-Holm equation (1.1) for $\rho=0$.

\section{General reduction scheme from AKNS system to the two-component Camassa-Holm equation}

Next, we perform the transformation

$$
\Psi \rightarrow \mathcal{U}(\theta, f) \Psi=\left[\begin{array}{l}
\varphi \\
\eta
\end{array}\right]
$$

on AKNS two-component $\Psi$ function from $(2.2) \cdot \mathcal{U}(\theta, f)$ stands for an orthogonal matrix:

$$
\mathcal{U}(\theta, f)=\Omega(\theta) \exp \left(-\frac{1}{2} f \sigma_{3}\right), \quad 0 \leq \theta \leq \frac{\pi}{2}
$$


where $\Omega(\theta)$ is given by

$$
\Omega(\theta)=\sigma_{3} e^{\mathrm{i} \theta \sigma_{2}}=\left[\begin{array}{cc}
\cos \theta & \sin \theta \\
\sin \theta & -\cos \theta
\end{array}\right]
$$

and $f$ is a function of $y$ and $s$, which is going to be determined below for each value of $\theta$.

Note that $\Omega^{-1}(\theta)=\Omega(\theta)$ and $\Omega(0)=\sigma_{3}, \Omega(\pi / 2)=\sigma_{1}$.

Taking a derivative with respect to $y$ and $s$ on both sides of (3.1) one gets

$$
\begin{aligned}
& {\left[\begin{array}{l}
\varphi \\
\eta
\end{array}\right]_{y}=\left(\mathcal{U}_{y} \mathcal{U}^{-1}+\mathcal{U}\left[\begin{array}{cc}
\lambda & q \\
r & -\lambda
\end{array}\right] \mathcal{U}^{-1}\right)\left[\begin{array}{l}
\varphi \\
\eta
\end{array}\right],} \\
& {\left[\begin{array}{l}
\varphi \\
\eta
\end{array}\right]_{s}=\left(\mathcal{U}_{s} \mathcal{U}^{-1}+\mathcal{U} D^{(-1)} \mathcal{U}^{-1}\right)\left[\begin{array}{l}
\varphi \\
\eta
\end{array}\right]}
\end{aligned}
$$

Thus, the flows of the new two-component function defined in (3.2) are governed by the gauge transformations of the AKNS matrices $\lambda \sigma_{3}+\mathcal{A}_{0}$ and $D^{(-1)}$, respectively. This ensures that the original AKNS compatibility condition (2.4) still holds for the rotated system defined by equations (3.4) and (3.5).

From equation (3.4) we derive that:

$$
\begin{aligned}
\lambda(\varphi \cos (2 \theta)+\eta \sin (2 \theta))= & \varphi_{y}+\frac{1}{2} \varphi\left(f_{y} \cos (2 \theta)-\sin (2 \theta)\left(q e^{-f}+r e^{f}\right)\right) \\
& +\eta\left(\frac{1}{2} f_{y} \sin (2 \theta)-r e^{f} \sin ^{2}(\theta)+q e^{-f} \cos ^{2}(\theta)\right) .
\end{aligned}
$$

Repeating derivation with respect to $y$ one more time yields

$$
\begin{aligned}
{\left[\begin{array}{l}
\varphi \\
\eta
\end{array}\right]_{y y} } & =\left[\left(\mathcal{U}_{y} \mathcal{U}^{-1}+\mathcal{U}\left[\begin{array}{cc}
\lambda & q \\
r & -\lambda
\end{array}\right] \mathcal{U}^{-1}\right)_{y}+\left(\mathcal{U}_{y} \mathcal{U}^{-1}+\mathcal{U}\left[\begin{array}{cc}
\lambda & q \\
r & -\lambda
\end{array}\right] \mathcal{U}^{-1}\right)^{2}\right]\left[\begin{array}{l}
\varphi \\
\eta
\end{array}\right] \\
& =\mathcal{U}\left[\begin{array}{cc}
\lambda^{2}-\lambda f_{y}+f_{y}^{2} / 4-f_{y y} / 2+q r & q_{y}-f_{y} q \\
r_{y}+f_{y} r & \lambda^{2}-\lambda f_{y}+f_{y}^{2} / 4+f_{y y} / 2+q r
\end{array}\right] \mathcal{U}^{-1}\left[\begin{array}{l}
\varphi \\
\eta
\end{array}\right] .
\end{aligned}
$$

For

$$
\left[\begin{array}{l}
\bar{\varphi} \\
\bar{\eta}
\end{array}\right]=\Omega(\theta)\left[\begin{array}{l}
\varphi \\
\eta
\end{array}\right]
$$

the result is

$$
\left[\begin{array}{c}
\bar{\varphi} \\
\bar{\eta}
\end{array}\right]_{y y}=\left[\begin{array}{cc}
\lambda^{2}-\lambda f_{y}+f_{y}^{2} / 4-f_{y y} / 2+q r & \left(q_{y}-f_{y} q\right) e^{-f} \\
\left(r_{y}+f_{y} r\right) e^{f} & \lambda^{2}-\lambda f_{y}+f_{y}^{2} / 4+f_{y y} / 2+q r
\end{array}\right]\left[\begin{array}{c}
\bar{\varphi} \\
\bar{\eta}
\end{array}\right]
$$

and shows in a transparent way that the condition for eliminating $\bar{\eta}$ from the equation for $\bar{\varphi}_{y y}$ requires $\left(q_{y}-f_{y} q\right) \exp (-f)=0$ or $q=\exp (f)$. Similarly, the condition for eliminating $\bar{\varphi}$ from the equation for $\bar{\eta}_{y y}$ requires $\left(r_{y}+f_{y} r\right) \exp (f)=0$ or $r=\exp (-f)$. Clearly these reductions reproduce results of the previous section.

To obtain a more general result we return to equation (3.7). Projecting on the $\varphi$-component in equation (3.7) gives

$$
\begin{aligned}
\varphi_{y y}= & \lambda^{2} \varphi-\lambda f_{y} \varphi+\left(\frac{1}{4} f_{y}^{2}+q r\right) \varphi+\left(-\frac{1}{2} f_{y} \cos (2 \theta)+\frac{1}{2}\left(q e^{-f}+r e^{f}\right) \sin (2 \theta)\right)_{y} \varphi \\
& +\left(-\frac{1}{2} f_{y} \sin (2 \theta)-q e^{-f} \cos ^{2} \theta+r e^{f} \sin ^{2} \theta\right)_{y} \eta .
\end{aligned}
$$


Next, we will eliminate $\eta$ in order to obtain an equation for the one-component variable $\varphi$. This is analogous to the calculation made below equation (2.11), where the first order two-component AKNS spectral problem was reduced to second order equation for the one-component function $\psi$. To accomplish the task we must choose $f$ so that the identity

$$
\frac{1}{2} f_{y} \sin (2 \theta)=r e^{f} \sin ^{2} \theta-q e^{-f} \cos ^{2} \theta+c_{0}
$$

holds, where $c_{0}$ is an integration constant. The identity (3.9) ensures that terms with $\eta$ drop out of equation (3.8).

Note, that for $\theta=\pi / 4$ and $c_{0}=0$ we recover identity $f_{y}=r \exp (f)-q \exp (-f)$ from $[11,19]$. For $\theta=0, c_{0}=1$ and $\theta=\pi / 2, c_{0}=-1$ we get, respectively, $q=\exp (f)$ and $r=\exp (-f)$ as in [20]. From now on we take $c_{0}=0$ as long as $0<\theta<\pi / 2$.

Let us shift a function $f$ by a constant term, $\ln (\tan \theta)$ :

$$
f \longrightarrow f_{\theta}=f+\ln (\tan \theta) \text {. }
$$

Then relation (3.9) can be rewritten for $0<\theta<\pi / 2$ as

$$
f_{\theta y}=r e^{f_{\theta}}-q e^{-f_{\theta}}
$$

which is of the same form as the relation found in reference [11]. It therefore appears that for all values of $\theta$ in the the $0<\theta<\pi / 2$ relation between function $f$ and AKNS variables $q$ and $r$ remains invariant up to shift of $f$ by a constant.

Now, we turn our attention back to equation (3.5) rewritten as

$$
\left[\begin{array}{l}
\varphi \\
\eta
\end{array}\right]_{s}=\mathcal{U}\left(-\frac{1}{2} f_{s} \sigma_{3}+\frac{1}{\lambda}\left[\begin{array}{cc}
A & B \\
C & -A
\end{array}\right]\right) \mathcal{U}^{-1}\left[\begin{array}{l}
\varphi \\
\eta
\end{array}\right]
$$

For the $\varphi$ component we find:

$$
\begin{aligned}
\varphi_{s}= & -\frac{1}{2} f_{s}(\varphi \cos (2 \theta)+\eta \sin (2 \theta))+\frac{1}{2 \lambda} \varphi\left(2 A \cos (2 \theta)+C e^{f} \sin (2 \theta)+B e^{-f} \sin (2 \theta)\right) \\
& +\frac{1}{\lambda} \eta\left(A \sin (2 \theta)+C e^{f} \sin ^{2} \theta-B e^{-f} \cos ^{2} \theta\right) .
\end{aligned}
$$

For $0<\theta<\pi / 2$ we choose

$$
B=\left(A-\frac{1}{4 \beta}\right) e^{f_{\theta}}, \quad C=-\left(A+\frac{1}{4 \beta}\right) e^{-f_{\theta}},
$$

which agrees with the determinant formula $A^{2}+B C=1 / 16 \beta^{2}$ and implies identities:

$$
\begin{aligned}
& 2 A-B e^{-f_{\theta}}+C e^{f_{\theta}}=0, \\
& B e^{-f_{\theta}}+C e^{f_{\theta}}=-\frac{1}{2 \beta} .
\end{aligned}
$$

The first of these identities, (3.14), ensures that the last three terms containing $\eta$ on the right hand side of equation (3.12) cancel.

Recall at this point relation (3.6). Simplifying this relation by invoking identity (3.9) and plugging it into equation (3.12) gives

$$
\varphi_{s}=-\frac{f_{s}}{2 \lambda} \varphi_{y}+\frac{1}{\lambda} \varphi\left(-\frac{1}{4} f_{s} f_{y} \cos (2 \theta)+\frac{f_{s}}{4} \sin (2 \theta)\left(q e^{-f}+r e^{f}\right)\right.
$$




$$
\left.+A \cos (2 \theta)+\frac{1}{2} B e^{-f} \sin (2 \theta)+\frac{1}{2} C e^{f} \sin (2 \theta)\right) .
$$

From (3.13) we find

$$
\begin{aligned}
& r e^{f_{\theta}}=\frac{C_{y}}{2 A} e^{f_{\theta}}=\frac{1}{2 A}\left(f_{y}\left(A+\frac{1}{4 \beta}\right)-A_{y}\right), \\
& q e^{-f_{\theta}}=-\frac{B_{y}}{2 A} e^{-f_{\theta}}=\frac{-1}{2 A}\left(f_{y}\left(A-\frac{1}{4 \beta}\right)+A_{y}\right)
\end{aligned}
$$

and therefore

$$
q e^{-f_{\theta}}+r e^{f_{\theta}}=\frac{f_{y}}{4 A \beta}-\frac{A_{y}}{A} .
$$

Due to the above relation and identity (3.15) equation (3.16) becomes

$$
\varphi_{s}=-\frac{f_{s}}{2 \lambda} \varphi_{y}+\frac{1}{\lambda} \varphi\left(-\frac{1}{4 \beta}\left(1-\frac{f_{s} f_{y}}{4 A}\right)-\frac{A_{y}}{A} \frac{f_{s}}{4}\right) .
$$

Taking derivative of (3.9) with respect to $s$ we find

$$
\frac{1}{2} f_{s y}=C e^{f_{\theta}}+B e^{-f_{\theta}}+\frac{1}{2} f_{s}\left(q e^{-f_{\theta}}+r e^{f_{\theta}}\right)=-\frac{1}{2 \beta}+\frac{f_{s} f_{y}}{8 A \beta}-\frac{f_{s} A_{y}}{2 A} .
$$

Thus equation (3.20) becomes

$$
\varphi_{s}=-\frac{f_{s}}{2 \lambda} \varphi_{y}+\frac{f_{s y}}{4 \lambda} \varphi .
$$

We now turn our attention to equation (3.8). The last term containing $\eta$ vanishes due to the identity (3.9). In addition it holds that

$$
\frac{f_{s y}}{2 f_{s}}+\frac{1}{2 \beta f_{s}}=-\frac{1}{2} f_{y} \cos (2 \theta)+\frac{1}{2}\left(q e^{-f}+r e^{f}\right) \sin (2 \theta)=\frac{1}{2}\left(q e^{-f_{\theta}}+r e^{f_{\theta}}\right)
$$

as follows from relations (3.19) and (3.21). Also, it holds from relations (3.17)-(3.18) that for $0<\theta<\pi / 2$ :

$$
r q=\left(\frac{f_{s y}}{2 f_{s}}+\frac{1}{2 \beta f_{s}}\right)^{2}-\frac{1}{4} f_{y}^{2}=g^{2}-f_{y}^{2} / 4,
$$

where

$$
g=\frac{f_{s y}}{2 f_{s}}+\frac{1}{2 \beta f_{s}} .
$$

Thus, the remaining constant (the ones which do not contain $\lambda$ ) terms on the right hand side of equation (3.8) are equal to

$$
\begin{gathered}
\frac{1}{4} f_{y}^{2}+q r+\left(-\frac{1}{2} f_{y} \cos (2 \theta)+\frac{1}{2}\left(q e^{-f}+r e^{f}\right) \sin (2 \theta)\right)_{y} \\
=\frac{1}{4} f_{y}^{2}+q r+\frac{1}{2}\left(q e^{-f_{\theta}}+r e^{f_{\theta}}\right)_{y}=g^{2}+g_{y} .
\end{gathered}
$$

Therefore, we can write equation (3.8) as:

$$
\varphi_{y y}=\left(\lambda^{2}-\lambda f_{y}-Q\right) \varphi, \quad Q=-g^{2}-g_{y}
$$

with $g$ given by (3.25). The above spectral problem together with equation (3.22) ensures via compatibility condition $\varphi_{y y s}-\varphi_{\text {syy }}=0$, that

$$
Q_{s}+\frac{1}{2} f_{y y} f_{s}+f_{y} f_{s y}=0
$$

holds. The latter is equivalent to the two-component Camassa-Holm equation (2.19). 


\section{The $\theta=0$ case and Bäcklund transformation between different solutions}

We now consider $\theta$ at the boundary of the $0<\theta<\pi / 2$ interval. For illustration we take $\theta=0$, the remaining case $\theta=\pi / 2$ can be analyzed in a similar way. Plugging $\theta=0$ into relation (3.26) we obtain

$$
\left.r q\right|_{\theta=0}=-\frac{1}{4} f_{y}^{2}+\frac{1}{2} f_{y y}+g^{2}+g_{y}=g^{2}-\frac{1}{4} f_{y}^{2}+\left(\frac{1}{2} f_{y}+g\right)_{y} .
$$

Comparing with relation (3.24) we get

$$
\left.r q\right|_{\theta=0}=\left.r q\right|_{\theta}+\left(\frac{1}{2} f_{y}+g\right)_{y}
$$

which describes a relation between the product $r q$ for zero and non-zero values of the angle $\theta$, with $\left.r q\right|_{\theta}$ being associated with $\theta$ within an interval $0<\theta<\pi / 2$.

Recall that $q=\exp (f)$ for $\theta=0$. It follows that $A=q_{s y} / 4 q=\left(f_{s y}+f_{s} f_{y}\right) / 4$ and equation (2.7) is equivalent to

$$
\left(\left.r q\right|_{\theta=0}\right)_{s}=\frac{1}{2}\left(f_{s y}+f_{s} f_{y}\right)_{y} .
$$

On the other hand, it follows from $(2.17)$ and $C=1 /\left(16 \beta^{2} B\right)-A^{2} / B$ that

$$
\left.r q\right|_{\theta=0}=\frac{1}{2}\left(f_{y y}-\frac{1}{2} f_{y}^{2}-\frac{f_{s y}^{2}}{2 f_{s}^{2}}+\frac{1}{2 \beta^{2} f_{s}^{2}}+\frac{f_{s y y}}{f_{s}}\right)
$$

and accordingly equation (4.2) is equivalent to the two-component Camassa-Holm equation (2.19).

From (3.18) one finds for $0<\theta<\pi / 2$ that:

$$
q=\mathcal{P}_{-}\left(f_{\theta}\right) e^{f_{\theta}}
$$

where

$$
\mathcal{P}_{ \pm}(f)= \pm \frac{1}{2} f_{y}+g= \pm \frac{f_{y}}{2}+\frac{f_{s y}}{2 f_{s}}+\frac{1}{2 \beta f_{s}} .
$$

Obviously $\mathcal{P}_{ \pm}\left(f_{\theta}\right)=\mathcal{P}_{ \pm}(f)$.

We are now ready to show that

$$
\bar{f}=f_{\theta}+\ln \left(\mathcal{P}_{-}\left(f_{\theta}\right)\right)=f_{\theta}+\ln \left(-\frac{f_{\theta y}}{2}+\frac{f_{\theta s y}}{2 f_{\theta s}}+\frac{1}{2 \beta f_{\theta s}}\right)
$$

satisfies the two-component Camassa-Holm equation (2.19) for any $f$ or $f_{\theta}$, which satisfies equation (2.19). For $0<\theta<\pi / 2$, it holds that $q=\exp (\bar{f})$ and therefore

$$
A=q_{s y} / 4 q=\left(\bar{f}_{s y}+\bar{f}_{s} \bar{f}_{y}\right) / 4=\left(f_{s y}+f_{s} f_{y}\right) / 4+\frac{f_{s} \mathcal{P}_{-y}+\mathcal{P}_{-y s}+\mathcal{P}_{-s} f_{y}}{4 \mathcal{P}_{-}} .
$$

We will now show that

$$
\left(\left.r q\right|_{\theta}\right)_{s}=\left(\left.r q\right|_{\theta=0}\right)_{s}-\left(\frac{1}{2} f_{y}+g\right)_{y s}=\frac{1}{2}\left(f_{s y}+f_{s} f_{y}\right)_{y}+\left(\frac{f_{s} \mathcal{P}_{-y}+\mathcal{P}_{-y s}+\mathcal{P}_{-s} f_{y}}{2 \mathcal{P}_{-}}\right)_{y}
$$


Using equation (4.2) one can easily show that equation (4.5) holds if the following relation

$$
-\left(f_{y}+\mathcal{P}_{-}\right)_{s}=\frac{f_{s} \mathcal{P}_{-y}+\mathcal{P}_{-y s}+\mathcal{P}_{-s} f_{y}}{2 \mathcal{P}_{-}}
$$

is true. We note that the above relation can be rewritten as

$$
\left(\mathcal{P}_{-}^{2}\right)_{s}+2 f_{y s} \mathcal{P}_{-}+f_{s} \mathcal{P}_{-y}+\mathcal{P}_{-s y}+\mathcal{P}_{-s} f_{y}=0 .
$$

The last equation is fully equivalent to the two-component Camassa-Holm equation (3.28) as can be seen by rewriting $Q$ from relation $(3.27)$ as $Q=-\left(\mathcal{P}_{-}+f_{y} / 2\right)^{2}-\left(\mathcal{P}_{-}+f_{y} / 2\right)_{y}$. This completes the proof for relation (4.5).

It follows from $(2.17)$ and $C=1 /\left(16 \beta^{2} B\right)-A^{2} / B$ that

$$
\left.r q\right|_{\theta}=\frac{1}{2}\left(\bar{f}_{y y}-\frac{1}{2} \bar{f}_{y}^{2}-\frac{\bar{f}_{s y}^{2}}{2 \bar{f}_{s}^{2}}+\frac{1}{2 \beta^{2} \bar{f}_{s}^{2}}+\frac{\bar{f}_{s y y}}{\bar{f}_{s}}\right) .
$$

Thus, due to (4.4) and (4.5) we have proved explicitly that

$$
\bar{f}=f+\ln \left(\tan \theta\left(-\frac{f_{y}}{2}+\frac{f_{s y}}{2 f_{s}}+\frac{1}{2 \beta f_{s}}\right)\right)=f_{\theta}+\ln \mathcal{P}_{-}\left(f_{\theta}\right)
$$

is a solution of a 2-component version of the Camassa-Holm equation. Thus the transformation

$$
f \rightarrow \bar{f}
$$

maps a solution $f$ of a 2-component version of the Camassa-Holm equation to a different solution $\bar{f}$. For example, let us consider, as in [21], the Camassa-Holm function:

$$
f(y, s)=\ln \frac{a_{1}^{(1)} a_{2}^{(1)} z_{1} e^{\frac{s}{2 z_{1}}+2 y z_{1}}+a_{1}^{(2)} a_{2}^{(2)} z_{2} e^{\frac{s}{2 z_{2}}+2 y z_{2}}}{\left(z_{2}-z_{1}\right) a_{1}^{(2)} a_{2}^{(1)}},
$$

where $a_{i}^{(j)}, i, j=1,2$ and $z_{1}$ and $z_{2}$ are constants. The function $f$ solves equation (2.19) for $\beta^{2}=1$. Then, as an explicit calculation verifies, the map $f \rightarrow \bar{f}$ with $\bar{f}$ given by expression (4.6) yields another solution of equation (2.19) for $\beta^{2}=1$ and $\theta \neq 0$.

For $\theta=\pi / 2$ we have $r=\exp (-f)$ and comparing with the result for $0<\theta<\pi / 2$ :

$$
r=\mathcal{P}_{+}\left(f_{\theta}\right) e^{-f_{\theta}},
$$

we get a Bäcklund transformation

$$
f \rightarrow f_{\theta}-\ln \left(\mathcal{P}_{+}\left(f_{\theta}\right)\right)=f_{\theta}-\ln \left(\frac{f_{\theta y}}{2}+\frac{f_{\theta s y}}{2 f_{\theta s}}+\frac{1}{2 \beta f_{\theta s}}\right) .
$$

Additional Bäcklund transformations can be obtained by comparing expressions for $q$ and $r$ variables in terms of $f$ for the boundary values of $\theta$.

We first turn our attention to the case of $\theta=0$ for which we have $q=\exp (f)$ and

$$
r=\frac{1}{2}\left(f_{y y}-\frac{1}{2} f_{y}^{2}-\frac{f_{s y}^{2}}{2 f_{s}^{2}}+\frac{1}{2 \beta^{2} f_{s}^{2}}+\frac{f_{\text {syy }}}{f_{s}}\right) e^{-f}=\left(\mathcal{P}_{+}^{2}-\mathcal{P}_{+} f_{y}+\mathcal{P}_{+y}\right) e^{-f} .
$$

From the AKNS equation (2.18) we see immediately that $f=\ln q$ must satisfy the 2-component Camassa-Holm equation (2.19). Note, in addition, that the AKNS equation (2.18) is still valid if we replace $q$ by $r$ and therefore

$$
f-\ln \left(\mathcal{P}_{+}^{2}-\mathcal{P}_{+} f_{y}+\mathcal{P}_{+y}\right)
$$

must satisfy the 2-component Camassa-Holm equation (2.19) as well. 
Next, for $\theta=\pi / 2$ we have $r=\exp (-f)$ and

$$
q=\frac{1}{2}\left(-f_{y y}-\frac{1}{2} f_{y}^{2}-\frac{f_{s y}^{2}}{2 f_{s}^{2}}+\frac{1}{2 \beta^{2} f_{s}^{2}}+\frac{f_{s y y}}{f_{s}}\right) e^{f}=\left(\mathcal{P}_{-}^{2}+\mathcal{P}_{-} f_{y}+\mathcal{P}_{-y}\right) e^{f} .
$$

Comparing expressions for $q$ and $r$ we find find that if $f$ is a solution of the 2-component Camassa-Holm equation (2.19) then so is also

$$
f+\ln \left(\mathcal{P}_{-}^{2}+\mathcal{P}_{-} f_{y}+\mathcal{P}_{-y}\right)
$$

To summarize we found the following Bäcklund maps

$$
f \rightarrow\left\{\begin{array}{l}
f_{\theta} \pm \ln \left(\mathcal{P}_{\mp}\left(f_{\theta}\right)\right), \quad f_{\theta}=f+\text { const } \\
f \pm \ln \left(\mathcal{P}_{\mp}^{2} \pm \mathcal{P}_{\mp} f_{y}+\mathcal{P}_{\mp y}\right) .
\end{array}\right.
$$

The top row lists maps between $\theta=0, \pi / 2$ cases and $\theta$ within the interval $0<\theta<\pi / 2$ [20]. The bottom row shows new maps derived for the $\theta=0$ and $\pi / 2$ cases only.

\section{Conclusions}

These notes describe an attempt to construct a general and universal formalism which would realize possible connections between the 2-component Camassa-Holm equation and AKNS hierarchy extended by a negative flow.

Construction yields gauge copies of an extended AKNS model connected by a continuous parameter (angle) $\theta$ taking values in an interval $0 \leq \theta \leq \pi / 2$. Eliminating one of two components of the $s l(2)$ wave function gives a second order non-linear partial differential equation for a single function $f$ of the two-component Camassa-Holm model. Functions $f$ corresponding to different values of $\theta$ in an interior of interval $0 \leq \theta \leq \pi / 2$ differ only by a trivial constant and fall into a class considered in [11]. Two remaining and separate cases correspond to $\theta$ equal to 0 and $\pi / 2$ and agree with a structure described in [20].

\section{Acknowledgements}

H.A. acknowledges partial support from Fapesp and IFT-UNESP for their hospitality. JFG and AHZ thank CNPq for a partial support.

[1] Aratyn H., Gomes J.F., Zimerman A.H., Integrable hierarchy for multidimensional Toda equations and topological-anti-topological fusion, J. Geom. Phys., 2003, V.46, 21-47, Erratum, J. Geom. Phys., 2003, V.46, 201-201, hep-th/0107056.

[2] Chodos A., Simple connection between conservation laws in the Korteweg-de Vries and sine-Gordon systems, Phys. Rev. D, 1980, V.21, 2818-2822.

[3] Olive D., Turok N., Local conserved densities and zero curvature conditions for Toda lattice field theories, Nuclear Phys., 1985, V.257, 277-301.

[4] Eguchi T., Yang S.K., Deformations of conformal field theories and soliton equations, Phys. Lett. B, 1989, V.224, 373-377.

[5] Tracy C.A., Widow H., Fredholm determinants and the $\mathrm{mKdV} /$ sinh-Gordon hierarchies, Comm. Math. Phys., 1996, V.179, 1-9, solv-int/9506006.

[6] Haak G., Negative flows of the potential KP-hierarchy, Trans. Amer. Math. Soc., 1996, V.348, 375-390.

[7] Dorfmeister J., Gradl H., Szmigielski J., Systems of PDEs obtained from factorization in loop groups, Acta Appl. Math., 1998, V.53, 1-58, solv-int/9801009.

[8] Aratyn H., Ferreira L.A., Gomes J.F., Zimerman A.H., The complex sine-Gordon equation as a symmetry flow of the AKNS hierarchy, J. Phys. A: Math. Gen., 2000, V.33, L331-L337, nlin.SI/0007002. 
[9] Camassa R., Holm D., An integrable shallow water equation with peaked solitons, Phys. Rev. Lett., 1993, V.71, 1661-1664, patt-sol/9305002.

[10] Fokas A., Fuchssteiner B., Symplectic structures, their Bäcklund transformation and hereditary symmetries, Phys. D, 1981, V.4, 47-66.

[11] Chen M., Liu S.Q., Zhang Y., A two-component generalization of the Camassa-Holm equation and its solutions, Lett. Math. Phys., 2006, V.75, 1-15, nlin.SI/0501028.

[12] Liu S.Q., Zhang Y., Deformations of semisimple bihamiltonian structures of hydrodynamic type, J. Geom. Phys., 2005, V.54, 427-453, math.DG/0405146.

[13] Falqui G., On a Camassa-Holm type equation with two dependent variables, J. Phys. A: Math. Gen., 2006, V.39, 327-342, nlin.SI/0505059.

[14] Shabat A.B., Martinez Alonso L., On the prolongation of a hierarchy of hydrodynamic chains, in New Trends in Integrability and Partial Solvability, Editors A.B. Shabat et al., Proceedings of the NATO Advanced Research Workshop (2002, Cadiz, Spain), NATO Sci. Ser. II Math. Phys. Chem., Vol. 132, Dordrecht, Kluwer Academic Publishers, 2004, 263-280.

[15] Adler V.E., Shabat A.B., Dressing chain for the acoustic spectral problem, nlin.SI/0604008.

[16] Ivanov R.I., Extended Camassa-Holm hierarchy and conserved quantities, Zeitschrift fur Naturforschung A, 2006, V.61, 133-138, nlin.SI/0601066.

[17] Martinez Alonso L., Shabat A.B., Hydrodynamic reductions and solutions of a universal hierarchy, Theoret. and Math. Phys., 2004, V.140, 1073-1085, nlin.SI/0312043.

[18] Shabat A.B., Universal solitonic hierarchy, J. Nonlinear Math. Phys., 2005, V.12, suppl. 1, 614-624.

[19] Aratyn H., Gomes J.F., Zimerman A.H., On negative flows of the AKNS hierarchy and a class of deformations of bihamiltonian structure of hydrodynamic type, J. Phys. A: Math. Gen., 2006, V.39, 1099-1114, nlin.si/0507062.

[20] Wu Ch.-Zh., On solutions of two-component Camassa-Holm system, J. Math. Phys., 2006, V.47, 083513, 11 pages.

[21] Aratyn H., van de Leur J., Clifford algebra derivations of tau functions for two-dimensional integrable models with positive and negative flows, nlin.SI/0605027. 